\title{
Branche umwirbt Fintechs
}

\author{
Die Banken, aber auch die Stadt Frankfurt am Main wollen für Finanz-Start- \\ ups attraktiver werden. Selbst der Regulierer zieht mit. Alle haben noch \\ Hausaufgaben zu erledigen, stellten sie auf der Euro Finance Week fest.
}

\author{
Bianca Baulig, Stefanie Hüthig
}

Auch Traditionsformate wie die Euro Finance Week müssen sich neuen Phänomenen anpassen. Das gelang dem Veranstalter Maleki Group mit der Euro Finance Tech Mitte November 2015. Statt Vorträgen reihten sich Diskussionen zu verschiedenen Aspekten des Themenkomplexes Fintech auf einem kleinen Podium in der Mitte des Saals aneinander. Mittags wurde den Start-ups Gelegenheit gegeben, sich in Pitches vorzustellen. Auch die Raumausstattung wich von dem ab, was Finanzexperten von Branchentreffen gewohnt sind. Die $\mathrm{Zu}-$ schauer nahmen statt in parlamentarischer Bestuhlung auf Barhockern, kleinen Holzbänken oder Sitzsäcken in Blau, Violett und einem grellen Grün Platz. Die etablierten Banker in ihren schwarzen oder blauen Anzügen trafen dort auf die Fintech-Gründer, die ihrerseits mittlerweile eine Art Dresscode entwickelt haben: Jeans mit Jackett, gerne auch zu Turnschuhen, auf jeden Fall ohne Krawatte.

Die Kreditwirtschaft ignoriert die Fintechs also nicht länger. Auch wenn Martin Deckert davor warnte, nur einen Abgesang auf die Banken anzustimmen. Deckert war Vorstand der UBS Deutschland und sprach bei der Veranstaltung in seiner Funktion als Managing Director. Es sei auch eine Frage, ob die Fintechs überleben, von denen viele kein Geld verdienten. Die etablierten Banken seien ein nicht zu unter-

\section{Kompakt}

- Kreditinstitute und Fintechs sind Wettbewerber, gleichzeitig aber auch Kooperationspartner.

- Um sich für die Zukunft zu rüsten, müssen Banken ihre Geschäftsmodelle anpassen.

- Gründer fordern bessere Rahmenbedingungen und mehr Dialog mit der Finanzaufsicht. schätzender Wettbewerber, „wenn die Maschine einmal läuft“. Deckert betonte, dass Kreditinstitute ihren Datenschatz künftig besser nutzen werden, ein Thema, das in den vergangenen Jahren aufgrund der Krise vernachlässigt worden sei.

Diese These vertrat auch Michael Arndt beim Kongress „Bankgeschäft im digitalen Umbruch“. Der Partner bei Bearing Point ist der Meinung, dass sich die Bedürfnisse der Kunden aus den vorhandenen Bankdaten zu wenig ablesen lassen. Um den sich ändernden Erwartungen der Verbraucher gerecht zu werden, müssten Geldhäuser neue Produkte entwickeln und ihr Angebot um nutzwertige Services anreichern. Es gehe darum, sich anpassungsfähig zu zeigen, nur so sei das Überleben am Markt gewährleistet. „Derzeit hat kein Kreditinstitut den Status, sich zurückzulehnen“, machte Arndt deutlich und schloss dabei ausdrücklich die Direktbanken ein.

\section{Community für Frankfurt am Main gründen}

Doch nicht nur für Banken und Sparkassen besteht Handlungsbedarf. Geht es um Gründungen, müssen sich auch die Stadt Frankfurt am Main und die Rhein-Main-Region Kritik gefallen lassen. Zwar schwärmen viele Experten von der Gründerszene Berlins. Andreas Hackethal, Professor für Finanzen und Dekan des Fachbereichs Wirtschaftswissenschaften der Goethe-Universität in Frankfurt, glaubt allerdings, dass der Mainmetropole nur „ein kleines bisschen“ beziehungsweise „die letzte Meile“ fehle, um zum führenden deutschen FintechHub zu werden. Vier oder fünf Experten sollten in Vollzeit an einer neuen Community arbeiten, die Fintechs und andere Player im Frankfurter Raum enger zusammenbringt, fordert Hackethal. Mitdiskutant Christian Hoppe, Geschäftsführer des Main Incubators und Head of Credit Solutions in der Mittelstandsbank der Commerzbank, bezifferte das dafür not- 


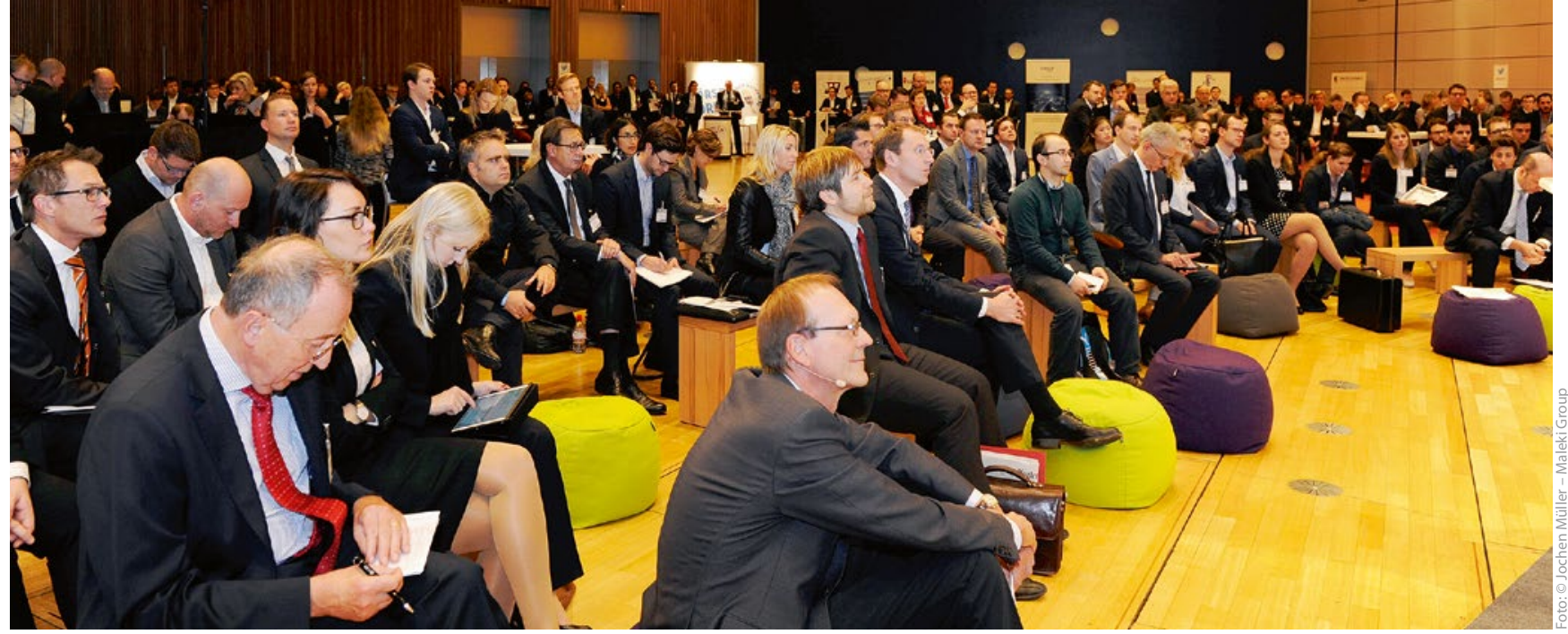

Banker trafen auf der Euro Finance Tech, einer Konferenz im Rahmen der Euro Finance Week, auf Gründer von Fintechs. Die Holzbänke und Sitzsäcke sollten den Aufbruch der Kreditwirtschaft in eine digitale und moderne Zukunft symbolisieren.

wendige Kapital auf 25 Millionen Euro. Diese Summe zu dem vorgeschlagenen Zweck zu beschaffen, sei auch in einer Stadt wie dem Finanzplatz Frankfurt schwierig. Er klagt, die RheinMain-Region sei „nicht laut genug“. Anders als Berlin, das viel ins Marketing stecke, schaffe Frankfurt und Umgebung die notwendigen Voraussetzungen - „und hört dann aber auf“.

Mit einem anderen Mangel hat Oliver Vins, Gründer und Vorstand von Vaamo, zu kämpfen. Das Fintech, das sich auf die Geldanlage spezialisiert hat, ist gut mit Backend-Programmierern ausgestattet. Entwickler für das Frontend fehlen Vins jedoch. Es sei mühsam, talentierte Entwickler von außerhalb von Frankfurt zu überzeugen, erklärt er. Christopher Oster, Chef des Online-Versicherungsmaklers Clark, verriet hingegen, dass es ihn aus persönlichen Gründen in die deutsche Finanzmetropole zog. Dort angekommen, suchte er über Zeitungsanzeigen nach Talenten und fand so binnen kurzer Zeit rund 200 Entwickler. Das wäre in Berlin nicht möglich gewesen, ist er sich sicher, da der War for Talents dort viel schärfer geführt werde. Oster kritisiert, dass in Frankfurt zu viel den Start-ups zugeschaut und zu wenig gegründet werde. Wenn Studenten in ihrem letzten Jahr an der Universität das Start-up nicht als Karriereoption sehen, würden sie auch nach ihrem Abschluss nicht den Weg in ein Fintech anstreben, meint der Clark-Chef.

\section{Wenig Dialog mit der Finanzaufsicht}

Ein weiterer Hemmschuh ist die Regulierung. Laut Hackethal sucht die Bundesanstalt für Finanzdienstleistungsaufsicht (Bafin) zwar das Gespräch mit den Fintechs. Hier handele es sich aber nicht um die letzte Meile, die zu gehen sei, die Behörde habe noch einen längeren Weg vor sich. Der Professor nannte als Gegenbeispiel die britische Finanzaufsicht, die Start-ups mit Beratung begleitet und Großbritannien sowie London als Fintech-Hub voranbringt. Dort werde sogar ge- meinsam mit den Fintechs ausprobiert, was beim Kunden funktioniert, um letztlich mehr Wettbewerb zu schaffen, beschrieb Hackethal die Situation. Und Hoppe glaubt, dass Frankfurt „definitiv“ zwei Jahre hinter London zurück sei.

Claire Kütemeier, Bereichsleiterin bei der Bafin, machte den Start-ups zunächst Hoffnung, ruderte dann aber etwas zurück. Es sei das Ziel der deutschen Finanzaufsicht, Fintechs zu helfen. So arbeite die Bafin gerade an einem Überblick, welche Lizenz für welches Geschäftsmodell notwendig sei. Allerdings gab Kütemeier zu bedenken, dass ihre Behörde in den Aufgaben und Befugnissen anders aufgestellt ist als ihr Pendant in Großbritannien. Eine derart weitgehende Unterstützung wie im angelsächsischen Raum könne die Bafin den Start-ups nicht bieten, aber „wir beantworten Fragen“ und „man kann mit uns reden“, versprach Kütemeier.

Der Dialog müsse schnell in Gang kommen, das sei wichtig für beide Seiten, drängt Levin Holle, Direktor im Bundesfinanzministerium. Denn wenn die Fintechs nicht in ein bereits bestehendes Regelwerk passen, müsse der Regulierer aktiv werden und Rahmenbedingungen für diese neuen Geschäftsmodelle schaffen. Auch für Gesetzgeber und Aufsicht seien die Digitalisierung und die damit einhergehende Entwicklungsgeschwindigkeit eine Herausforderung. Die bisherigen Regeln, erklärte Holle, seien für papierbasierte Geschäfte gestaltet worden. Wie Kütemeier betonte Holle, an Lösungen zu arbeiten.
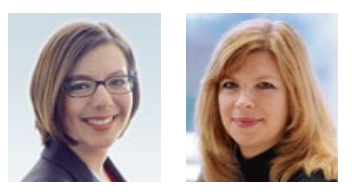

Autorinnen: Stefanie Hüthig ist BANKMAGAZIN-Chefredakteurin und schreibt am liebsten über Digitalisierung. Bianca Baulig ist Redakteurin beim BANKMAGAZIN. Ihre Schwerpunkte liegen auf den Themen Vertrieb und Strategie. 